\title{
Two-year follow-up of ranibizumab combined with photodynamic therapy for polypoidal choroidal vasculopathy
}

This article was published in the following Dove Press journal:

Clinical Ophthalmology

5 October 2012

Number of times this article has been viewed

\author{
Rei Nemoto ${ }^{1,2}$ \\ Masahiro Miura ${ }^{1,2}$ \\ Takuya Iwasaki ${ }^{1,2}$ \\ Hiroshi Goto ${ }^{2}$ \\ 'Department of Ophthalmology, \\ Tokyo Medical University, Ibaraki \\ Medical Center, Ami, Ibaraki, Japan; \\ ${ }^{2}$ Department of Ophthalmology, Tokyo \\ Medical University, Nishi-Shinjuku, \\ Tokyo, Japan
}

Purpose: We evaluated the 2-year efficacy of combined intravitreal ranibizumab (IVR) treatment and photodynamic therapy (PDT) for treatment-naïve polypoidal choroidal vasculopathy (PCV).

Patients and methods: Twenty-two eyes of 22 Japanese patients with treatment-naïve PCV were prospectively recruited. All eyes had angiographic features of PCV according to indocyanine green angiography. The initial combination treatment regimen included a session of PDT with IVR. A total of three consecutive IVR treatments were given at 4-week intervals. Eyes were retreated with IVR or PDT at specific times. We evaluated the mean visual acuity and mean central retinal thickness (CRT) at 3, 6, 9, 12, 18, and 24 months after initial treatment.

Results: At month 9, visual acuity had improved by 5.7 letters $(P=0.10)$. Subsequently, mean visual acuity gradually decreased, and the difference from baseline was diminished to 2.9 letters at 24 months $(P=0.43)$. Mean CRT was significantly decreased from baseline over the 24-month follow-up $(P<0.05)$.

Conclusion: With PDT combined with IVR for PCV, visual acuity improved during year 1, but the benefit decreased in year 2 .

Keywords: photodynamic therapy, polypoidal choroidal vasculopathy, ranibizumab

\section{Introduction}

Polypoidal choroidal vasculopathy $(\mathrm{PCV})$ is characterized by numerous recurrent, bilateral, asymmetric, serosanguineous detachments in the retinal pigment epithelium. ${ }^{1}$ The incidence of PCV is high in Asian populations, ${ }^{2}$ especially in the Japanese population. ${ }^{3}$ Without treatment, eyes with PCV may develop recurrent episodes of hemorrhage and exudative maculopathy, causing visual loss. ${ }^{4}$

Several treatment strategies have been reported for $\mathrm{PCV}^{2}$ Among them, photodynamic therapy (PDT) with verteporfin (Novartis Pharma AG, Basel, Switzerland) ${ }^{5,6}$ and intravitreal injection of anti-vascular endothelial growth factor (VEGF), including ranibizumab (Lucentis; Genentech, South San Francisco, CA, USA) ${ }^{7,8}$ and bevacizumab (Avastin; Genentech), ${ }^{8,9}$ have shown favorable results. However, several problems have been reported with these treatments. Serious complications after PDT for PCV were reported, such as massive subretinal hemorrhage, ${ }^{10-12}$ retinal pigment epithelium tearing, ${ }^{10}$ and retinal atrophy. ${ }^{12}$ With PDT, polypoidal lesions regressed in $86 \%$ to $95 \%$ of cases; ${ }^{5,13,14}$ however, polypoidal lesions were recurrent in $59 \%$ to $77 \%$ of the cases. $^{10,12}$ With anti-VEGF therapy, polypoidal lesions regressed in only $23 \%$ to $40 \%$ of the cases; ${ }^{7-9}$ hence, its long-term efficacy is not clear.
Correspondence: Masahiro Miura Department of Ophthalmology, Tokyo Medical University, Ibaraki Medical Center 3-20-I Chuo,

Ami, Inashiki, Ibaraki 300395, Japan

$\mathrm{Tel}+8 \mid 29887$ | | |6 |

Fax +8I 298877656

Email m-miura@tokyo-med.ac.jp 
Another treatment choice is a combination therapy of PDT and anti-VEGF drugs in expectation of a cumulative effect. PDT occluded polypoidal lesions and anti-VEGF drugs reduced fluid leakage from vascular lesions. However, little information is available regarding the long-term efficacy of this combination. In the 2-year results for the combination of PDT and intravitreal bevacizumab injection, regression of the treatment effect was reported in year $2 .{ }^{15,16}$ For PDT with intravitreal injection of ranibizumab (IVR), follow-up time was limited to only one year; ${ }^{17,18}$ hence, long-term results for combination of PDT with IVR are not clear. In this study, we evaluated the 2-year results of PDT with IVR treatment, and evaluated the efficacy and safety of this combination therapy.

\section{Material and methods}

We prospectively evaluated 22 eyes of 22 Japanese patients with treatment-naïve PCV and visual disturbance. All eyes were treated with PDT combined with IVR from March 2009 to April 2012 at Tokyo Medical University, Ibaraki Medical Center. All eyes had a minimum follow-up period of 24 months after the initial treatment. The clinical diagnosis of PCV was made by identification of polypoidal lesions with indocyanine green angiography (ICGA). Exclusion criteria were: (1) history of treatment for PCV or age-related macular degeneration and (2) presence of other retinal diseases, such as diabetic maculopathy or retinal vascular occlusion. This study was performed according to the tenets of the Declaration of Helsinki and was approved by the Institutional Review Boards of Tokyo Medical University, Ibaraki Medical Center. Informed consent for the examination was obtained from all patients. At each visit, all patients received a comprehensive ophthalmologic examination, including best-corrected visual acuity testing with the early treatment diabetic retinopathy study chart at 2 meters, indirect ophthalmoscopy, slit-lamp biomicroscopy with a contact lens, fundus photography, and optical coherence tomography (OCT: 3D OCT-2000;Topcon, Tokyo, Japan). Central retinal thickness (CRT) was measured using an OCT B-scan image.

The initial combination treatment regimen comprised a session of PDT after an intravitreal injection of $0.5 \mathrm{mg}$ ranibizumab. A total of three consecutive IVR treatments were given at 4-week intervals. Intravitreal injection was performed in a sterile manner and prophylactic topical antibiotics were applied for 3 days after the injection. At 4 days after the initial IVR treatment, PDT was performed using a 689-nm diode laser unit (Visulas PDT system 690S; Carl Zeiss, Dublin, California, USA) after an injection of verteporfin (mean: $10.5 \mathrm{mg}$ ), according to PDT guidelines for age-related macular degeneration. ${ }^{19}$ The greatest linear dimension was determined using fluorescein and indocyanine green angiograms, and comprised both polypoidal lesions and branching vascular network. The mean greatest linear dimension at baseline was $2753 \mu \mathrm{m}$ (standard deviation [SD]: $1340 \mu \mathrm{m}$ ). After the initial treatment regimen, follow-up evaluations were scheduled at one-month intervals. Fluorescein and indocyanine green angiography were performed on each patient using a confocal scanning laser ophthalmoscope (F-10, Nidek, Gamagori, Japan) before and at 3 months after treatment. Additional angiography was performed as necessary.

Retreatment was considered based on the protocol in the PrONTO study. ${ }^{20}$ An additional intravitreal injection was given if any of the following changes were observed: (1) visual acuity loss of at least five letters with OCT evidence of fluid in the macula, (2) an increase in OCT central retinal thickness of at least $100 \mu \mathrm{m}$, (3) enlargement of a pigment epithelial detachment, (4) new macular hemorrhages; or (5) persistent fluid on OCT one month after the previous treatment. Additional PDT was considered when intravitreal injection of ranibizumab was minimally effective and polypoidal lesions with exudative changes were detected in ICGA.

Visual acuity scores and central retinal thickness at 3, 6, 9, 12,18 , and 24 months after initial treatment were compared with baseline scores using a Wilcoxon signed-rank test. Statistical significance was defined as $P<0.05$. Statistical software (Statview 5.0; SAS Institute, Cary, NC, USA) was used for statistical analyses.

\section{Results}

This study included 22 eyes of 22 patients (19 males, 3 females), ranging in age from 60 years to 86 years (mean: 73.2 years) that underwent PDT combined with IVR treatment. Of the 22 eyes, subfoveal findings were polypoidal lesions in 18 eyes and a branching vascular network in 4 eyes. At baseline, best-corrected visual acuity using Landolt $\mathrm{C}$ charts ranged from 0.04 to 0.8 (median 0.4). At baseline, mean visual acuity letter score was 49.8 letters (standard error [SE]: 4.3) and mean CRT was $255.3 \mu \mathrm{m}$ (SE: 25.0). During the 24-month follow up, the total number of PDT treatments ranged from 1 to 4 (mean [SD]: 1.5 [1.0]), and the total number of IVR treatments ranged from 3 to 12 (mean [SD]: 7.3 [3.1]). A mean of 1.2 PDT and 5.4 IVR treatments were performed during the first year. A mean of 0.3 PDT and 1.9 IVR treatments were performed during the second year. 
Figure 1 shows the changes in mean visual acuity. At month 9 , visual acuity was improved by 5.7 letters $(P=0.10)$. Subsequently, mean visual acuity gradually decreased, and the difference from baseline was diminished to 2.9 letters at 24 months $(P=0.43)$. At month 24,10 eyes $(45 \%)$ gained at least 5 lines compared with baseline and 6 eyes (27\%) lost at least 5 lines from baseline (Table 1). Figure 2 shows changes in the mean CRT. Mean CRTs were decreased by $99.1 \mu \mathrm{m}$ and $113.7 \mu \mathrm{m}$ at 12 months and 24 months, respectively. Mean CRTs were significantly decreased from baseline through the 24 months of follow-up $(P<0.05)$.

In ICGA images at 3 months, polypoidal lesions were reduced in all eyes and disappeared completely in 16 eyes (72.7\%). Branching vascular networks persisted after treatment in all eyes (Figure 3). During the follow-up period, recurrence of polypoidal lesions was confirmed in a total of 14 eyes $(70 \%)$. Recurrence occurred before 12 months in two eyes and later in twelve eyes. Among the eyes with recurrence, three eyes showed extensive subretinal hemorrhage larger than a 2-disk diameter (Figure 3). Subretinal hemorrhage in each case occurred at 5, 16, and 21 months after initial treatment. In each eye, a retinal pigment epithelial tear was not detected with OCT or in ICGA images. Visual acuity scores at 24 months in each case were decreased by 48,13 , and 15 letters from baseline, respectively. The mean total number of IVR treatments with extensive subretinal hemorrhage was 9.3 (SD: 2.9) and the mean total number of IVR treatments without extensive subretinal hemorrhage was 7.0 (SD: 3.8). There was no significant difference in the total number of IVR treatments between the eyes with or without extensive subretinal hemorrhage ( $P=0.22$ : unpaired $t$-test). The mean greatest linear dimension at baseline in the eyes with extensive subretinal hemorrhage was $3464 \mu \mathrm{m}$ (SD: $288 \mu \mathrm{m}$ ) and

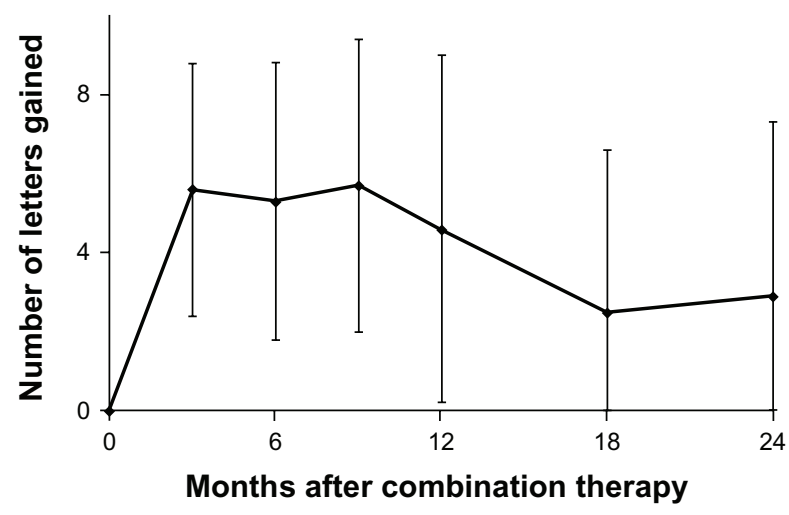

Figure I Mean change in visual acuity scores (letters) through 24 months for eyes with polypoidal choroidal vasculopathy treated with combined photodynamic therapy and intravitreal ranibizumab injection.

Note: Vertical lines are I standard error of the mean.
Table I Distribution of visual acuity changes from month 12 through month 24

\begin{tabular}{lll}
\hline Change in visual acuity from baseline & Month I2 & Month 24 \\
\hline$\geq 5$ line increase & $10(45 \%)$ & $10(45 \%)$ \\
$<5$ line increase to $>5$ line decrease & $8(36 \%)$ & $6(27 \%)$ \\
$\geq 5$ line decrease & $4(18 \%)$ & $6(27 \%)$ \\
\hline
\end{tabular}

the mean greatest linear dimension at baseline in the eyes without extensive subretinal hemorrhage was $2641 \mu \mathrm{m}$ (SD: $1410 \mu \mathrm{m})$. There was no significant difference in the greatest linear dimension between the eyes with or without extensive subretinal hemorrhage ( $P=0.33$ : unpaired $t$-test).

\section{Discussion}

Several studies have evaluated the efficacy of combined PDT and intravitreal bevacizumab injection for PCV. In these studies, significant improvement of visual acuity was obtained in the first year; however, treatment efficacy was decreased in year $2 .{ }^{15,16}$ Our study on combined PDT and IVR treatment showed similar results to combined PDT with intravitreal bevacizumab injection. These results suggest the chronic and refractory nature of PCV with combination therapies. In the studies of PDT for PCV, several factors, such as recurrence of polypoidal lesions, ${ }^{10,12}$ subretinal hemorrhage, ${ }^{10-12}$ retinal pigment epithelium tearing, ${ }^{10}$ or retinal atrophy, ${ }^{12}$ were considered to be factors reducing treatment efficacy. In our case series, a recurrence of polypoidal lesions or subretinal hemorrhage was a possible reason for the reduction in treatment efficacy.

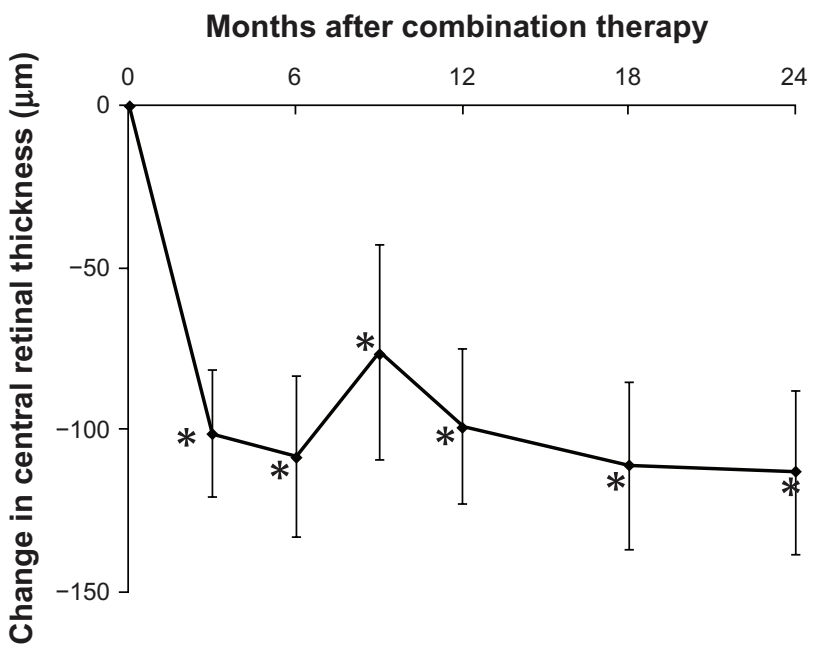

Figure 2 Mean change in central retinal thickness through 24 months for eyes with polypoidal choroidal vasculopathy treated with combined photodynamic therapy and intravitreal ranibizumab injection.

Notes: Vertical lines are I standard error of the mean. Central retinal thickness was significantly decreased from baseline through 24 months $(* P<0.05$, Wilcoxon signed-rank test). 

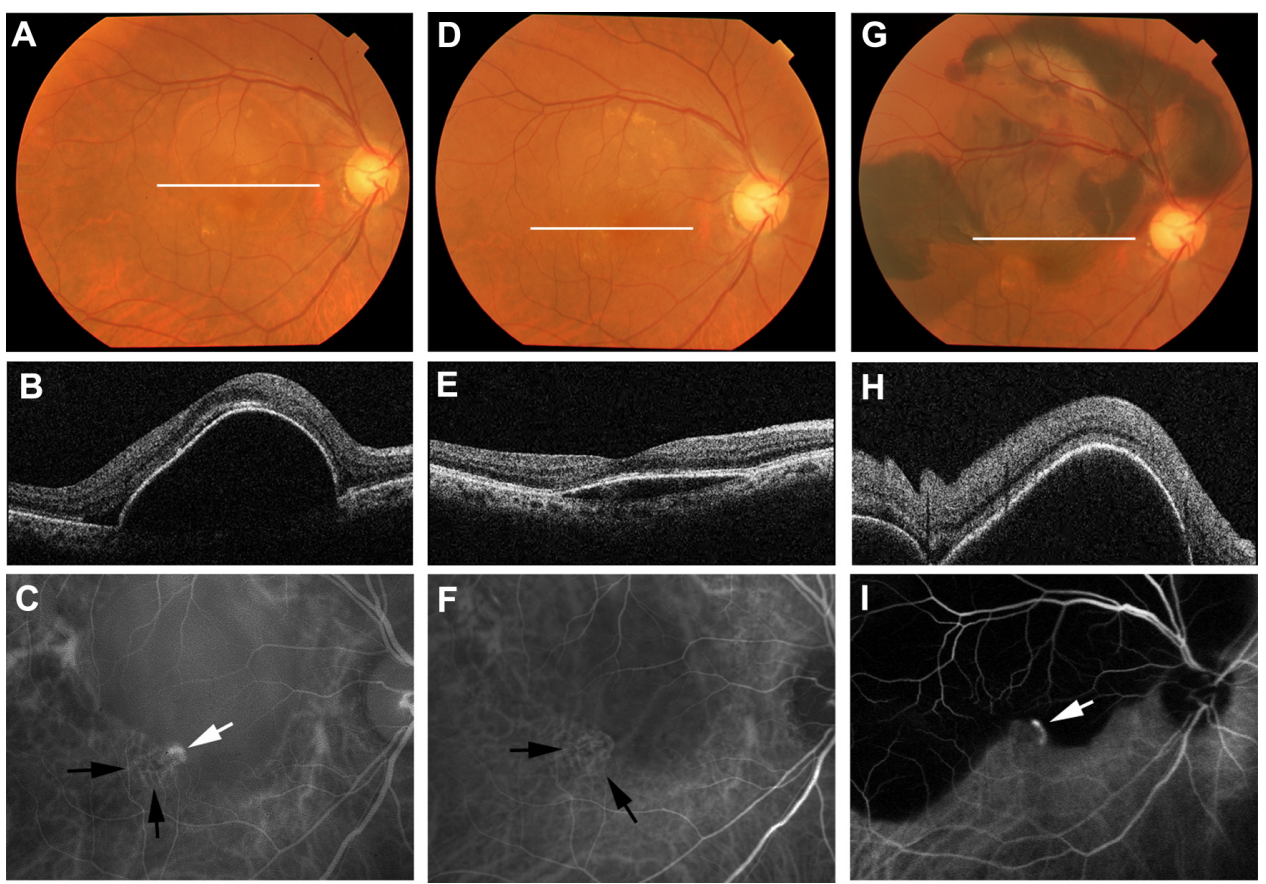

Figure 3 Right eye of a 74-year old male with subretinal hemorrhage after combined PDT and IVR treatment for PCV. White lines in each color fundus photograph (A, D and $\mathbf{G}$ ) show the scanning line of the OCT image at each time point. Baseline OCT (B) shows retinal pigment epithelial detachment on the macula. Baseline visual acuity was 0.7. ICGA (C) shows a polypoidal lesion (white arrow) and branching vascular network (black arrows). Three months after initial treatment, the retinal pigment epithelial detachment was reduced in OCT (E). Visual acuity was improved to 0.9. In ICGA (F), the branching vascular network persisted (black arrows), despite the disappearance of polypoidal lesions. Five months after initial treatment, color fundus photography $(\mathbf{G})$ and OCT $(\mathbf{H})$ showed massive hemorrhagic retinal pigment epithelial detachment in the macula. Visual acuity was decreased to 0.3. ICGA (I) shows recurrence of polypoidal lesions (white arrow).

Abbreviations: ICGA, indocyanine green angiography; IVR, intravitreal ranibizumab; OCT, optical coherence tomography; PDT, photodynamic therapy; PCV, polypoidal choroidal vasculopathy

In previous studies, both PDT monotherapy and combined PDT with anti-VEGF injection showed favorable results for the regression of polypoidal lesions. ${ }^{5,13,14,18,21-24}$ In the first year, regression of polypoidal lesions was observed in $86 \%$ to $95 \%$ of cases for PDT monotherapy ${ }^{5,13,14}$ and $72 \%$ to $100 \%$ for combined PDT with anti-VEGF injection. ${ }^{18,21-23}$ However, at the 1-year follow-up, recurrence of polypoidal lesions occurred in $5.6 \%$ to $16 \%$ of the cases with PDT monotherapy, ${ }^{5,14,22}$ and $10 \%$ to $29 \%$ of the cases with combined PDT and anti-VEGF therapy. ${ }^{18,22}$ The recurrence rate was increased to $64 \%$ of the cases at the 2 -year follow up for PDT monotherapy. ${ }^{25}$ The recurrence rate in our study was similar to that found in the 2-year results for PDT monotherapy. Several factors may be related to the reoccurrence of polypoidal lesions. One possible reason is the persistence of the branching vascular network. In our study, the branching vascular network persisted after combined PDT with IVR treatment, despite a reduction of polypoidal lesions as previously reported for PDT monotherapy ${ }^{25}$ or combined PDT with intravitreal bevacizumab injection. ${ }^{17}$ A branching vascular network was considered to be an affinity of choroidal neovascularization in exudative age-related macular degeneration. ${ }^{26,27}$ The presence of a branching vascular network may indicate the persistence of a main vascular component of PCV and may induce the frequent recurrence of polypoidal lesions. Further investigation is required to evaluate the mechanism of the recurrence of polypoidal lesions.

Another possible reason for the reduction of efficacy was massive subretinal hemorrhage. In our case series, three eyes showed massive subretinal hemorrhage and visual acuity at 24 months in each case was notably decreased from baseline. In previous studies, subretinal hemorrhage occurred in $7 \%$ to $30 \%$ of the cases with PDT monotherapy $y^{6,10,11}$ and in $3 \%$ to $17.7 \%$ of the cases with combined PDT and anti-VEGF injection. ${ }^{17,23,28}$ In these studies, PDT was considered a possible reason for subretinal hemorrhage. However, subretinal hemorrhage is a common complication in the natural history of $\mathrm{PCV}^{4}{ }^{4}$ Comparison with the natural history is important for evaluating the causative factors of massive subretinal hemorrhage; however, a comparison study with natural history is ethically difficult in medical practice. Considering alternative methods, comparison with anti-VEGF injection monotherapy is important for confirming the association of PDT with subretinal hemorrhage. However, long-term results for anti-VEGF injection monotherapy for PCV are not 
currently available. Further studies are required to evaluate the association of PDT with subretinal hemorrhage.

In previous studies, there were some variations in the treatment regimens for combination IVR treatment and PDT for PCV. In the EVEREST study, ${ }^{24}$ both PDT and IVR treatments were conducted on same day. ${ }^{20}$ In other studies, PDT was performed at 4 days to 7 days after initial IVR treatment. ${ }^{16,17}$ In this study, there was a 4-day interval between PDT and initial IVR treatment to examine the postoperative complications of IVR treatment. With regard to the number of IVR treatments, a total of three consecutive IVR treatments were given at 4-week intervals, in this study and the EVEREST study. ${ }^{24}$ In another study, only one session of IVR treatment was performed with PDT. ${ }^{17}$ These variations in treatment regimens may be related to the cumulative effect of IVR treatment and PDT. Further investigations are required to establish the optimal combination regime for treating PCV.

In conclusion, combined PDT with IVR treatment has a certain effect on PCV for up to 2 years. Careful follow-up, particularly for accompanying subretinal hemorrhage and the recurrence of polypoidal lesions, is essential for therapeutic management of PCV. A shortcoming of our study was the small number of cases and absence of comparisons with other treatment modalities. Further investigations are required to establish the optimal treatment strategy for PCV.

\section{Conclusion}

In conclusion, PDT combined with IVR treatment for PCV showed improved visual acuity during year 1 . However, the benefit of visual acuity was decreased by year 2 .

\section{Acknowledgments/disclosure}

The authors have no conflicts of interest, or financial interest, in this work. This project was supported in part by KAKENHI (24592682).

\section{References}

1. Yannuzzi LA, Sorenson J, Spaide RF, Lipson B. Idiopathic polypoidal choroidal vasculopathy (IPCV). Retina. 1990;10(1):1-8.

2. Laude A, Cackett PD, Vithana EN, et al. Polypoidal choroidal vasculopathy and neovascular age-related macular degeneration: same or different disease? Prog Retin Eye Res. 2009;29(1):19-29.

3. Maruko I, Iida T, Saito M, Nagayama D, Saito K. Clinical characteristics of exudative age-related macular degeneration in Japanese patients. Am J Ophthalmol. 2007;144(1):15-22.

4. Uyama M, Wada M, Nagai Y, et al. Polypoidal choroidal vasculopathy: natural history. Am J Ophthalmol. 2002;133(5):639-648.

5. Gomi F, Ohji M, Sayanagi K, et al. One-year outcomes of photodynamic therapy in age-related macular degeneration and polypoidal choroidal vasculopathy in Japanese patients. Ophthalmology. 2008;115(1): 141-146.
6. Hikichi T, Ohtsuka H, Higuchi M, et al. Factors predictive of visual acuity outcomes 1 year after photodynamic therapy in Japanese patients with polypoidal choroidal vasculopathy. Retina. 2011;31(5):857-865.

7. Hikichi T, Higuchi M, Matsushita T, et al. One-year results of three monthly ranibizumab injections and as-needed reinjections for polypoidal choroidal vasculopathy in Japanese patients. Am J Ophthalmol. 2012;154(1):117-124.

8. Cho HJ, Kim JW, Lee DW, Cho SW, Kim CG. Intravitreal bevacizumab and ranibizumab injections for patients with polypoidal choroidal vasculopathy. Eye. 2012;26(3):426-433.

9. Cheng CK, Peng CH, Chang CK, Hu CC, Chen LJ. One-year outcomes of intravitreal bevacizumab (avastin) therapy for polypoidal choroidal vasculopathy. Retina. 2011;31(5):846-856.

10. Leal S, Silva R, Figueira J, et al. Photodynamic therapy with verteporfin in polypoidal choroidal vasculopathy: results after 3 years of follow-up. Retina. 2010;30(8):1197-1205.

11. Hirami Y, Tsujikawa A, Otani A, et al. Hemorrhagic complications after photodynamic therapy for polypoidal choroidal vasculopathy. Retina. 2007;27(3):335-341.

12. Akaza E, Yuzawa M, Mori R. Three-year follow-up results of photodynamic therapy for polypoidal choroidal vasculopathy. Jpn J Ophthalmol. 2011;55(1):39-44.

13. Chan WM, Lam DS, Lai TY, et al. Photodynamic therapy with verteporfin for symptomatic polypoidal choroidal vasculopathy: one-year results of a prospective case series. Ophthalmology. 2004;111(8):1576-1584.

14. Akaza E, Yuzawa M, Matsumoto Y, Kashiwakura S, Fujita K, Mori R. Role of photodynamic therapy in polypoidal choroidal vasculopathy. Jpn J Ophthalmol. 2007;51(4):270-277.

15. Kim M, Kim K, Kim do G, Yu SY, Kwak HW. Two-year results of photodynamic therapy combined with intravitreal anti-vascular endothelial growth factor for polypoidal choroidal vasculopathy. Ophthalmologica. 2011;226(4):205-213.

16. Lee YA, Yang CH, Yang CM, et al. Photodynamic therapy with or without intravitreal bevacizumab for polypoidal choroidal vasculopathy: two years of follow-up. Am J Ophthalmol. In press.

17. Tomita K, Tsujikawa A, Yamashiro K, et al. Treatment of polypoidal choroidal vasculopathy with photodynamic therapy combined with intravitreal injections of ranibizumab. Am J Ophthalmol. 2012;153(1): 68-80.

18. Lee YH, Lee EK, Shin KS, Lee KM, Kim JY. Intravitreal ranibizumab combined with verteporfin photodynamic therapy for treating polypoidal choroidal vasculopathy. Retina. 2011;31(7):1287-1293.

19. Barbazetto I, Burdan A, Bressler NM, et al. Photodynamic therapy of subfoveal choroidal neovascularization with verteporfin: fluorescein angiographic guidelines for evaluation and treatment - TAP and VIP report No 2. Arch Ophthalmol. 2003;121(9):1253-1268.

20. Lalwani GA, Rosenfeld PJ, Fung AE, et al. A variable-dosing regimen with intravitreal ranibizumab for neovascular age-related macular degeneration: year 2 of the PrONTO Study. Am J Ophthalmol. 2009;148(1):43-58.

21. Ruamviboonsuk P, Tadarati M, Vanichvaranont S, Hanutsaha P, Pokawattana N. Photodynamic therapy combined with ranibizumab for polypoidal choroidal vasculopathy: results of a 1-year preliminary study. Br J Ophthalmol. 2010;94(8):1045-1051.

22. Kim SJ, Yu HG. Efficacy of combined photodynamic therapy and intravitreal bevacizumab injection versus photodynamic therapy alone in polypoidal choroidal vasculopathy. Retina. 2011;31(9):1827-1834.

23. Sato T, Kishi S, Matsumoto H, Mukai R. Combined photodynamic therapy with verteporfin and intravitreal bevacizumab for polypoidal choroidal vasculopathy. Am J Ophthalmol. 2010;149(6): 947-954.

24. Koh A, Lee WK, Chen LJ, et al. EVEREST STUDY: Efficacy and Safety of Verteporfin Photodynamic Therapy in Combination with Ranibizumab or Alone Versus Ranibizumab Monotherapy in Patients with Symptomatic Macular Polypoidal Choroidal Vasculopathy. Retina. 2012;32(8):1453-1464. 
25. Akaza E, Mori R, Yuzawa M. Long-term results of photodynamic therapy of polypoidal choroidal vasculopathy. Retina. 2008;28(5):717-722.

26. Miura M, Makita S, Iwasaki T, Yasuno Y. Three-dimensional visualization of ocular vascular pathology by optical coherence angiography in vivo. Invest Ophthalmol Vis Sci. 2011;52(5):2689-2695.

27. Costa RA, Navajas EV, Farah ME, Calucci D, Cardillo JA, Scott IU. Polypoidal choroidal vasculopathy: angiographic characterization of the network vascular elements and a new treatment paradigm. Prog Retin Eye Res. 2005;24(5):560-586.
28. Gomi F, Sawa M, Wakabayashi T, Sasamoto Y, Suzuki M, Tsujikawa M. Efficacy of intravitreal bevacizumab combined with photodynamic therapy for polypoidal choroidal vasculopathy. Am J Ophthalmol. 2010; 150(1):48-54.

\section{Publish your work in this journal}

Clinical Ophthalmology is an international, peer-reviewed journal covering all subspecialties within ophthalmology. Key topics include: Optometry; Visual science; Pharmacology and drug therapy in eye diseases; Basic Sciences; Primary and Secondary eye care; Patient Safety and Quality of Care Improvements. This journal is indexed on

\section{Dovepress}

PubMed Central and CAS, and is the official journal of The Society of Clinical Ophthalmology (SCO). The manuscript management system is completely online and includes a very quick and fair peer-review system, which is all easy to use. Visit http://www.dovepress.com/ testimonials.php to read real quotes from published authors. 